\title{
Heat Loss by Helicity Injection in Spheromaks
}

\author{
T.K. Fowler
}

March 17, 1994

This is an informal report intended primarily for internal or limited external distribution. The opinions and conclusions stated are those of the author and may or may not be those of the Laboratory.

Work performed under the auspices of the U.S. Department of Energy by the Lawrence Livermore National Laboratory under Contract W-7405-Eng-48. 


\section{DISCLAIMER}

This document was prepared as an acccount of work sponsored by an agency of the United States Government. Neither the United States Government nor the University of California nor any of their employees, makes any warranty, express or implied, or assumes any legal liability or responsibility for the accuracy, completeness, or usefulness of any information, apparatus, product, or process disclosed, or represents that its use would not infringe privately own rights. Reference herein to any specific commercial products, process, or service by trade name, trademark, manufacturer, or otherwise, does not necessarily constitute or imply its endorsement, recommendation, or favoring by the United States Government or the University of California. The views and opinions of authors expressed herein do not necessarily state or reflect those of the United States Government or the University of California, and shall not be used for advertising or product endorsement purposes.

This report has been reproduced directly from the best available copy.

Available to DOE and DOE contractors from the Office of Scientific and Technical Information P.O. Box 62, Oak Ridge, TN 37831

Prices available from (615) 576-8401, FIS 626-8401

Available to the public from the National Technical Information Service

U.S. Department of Commerce 5285 Port Royal Rd.,

Springfield, VA 22161 


\section{DISCLAIMER}

Portions of this document may be illegible in electronic image products. Images are produced from the best available original document. 


\title{
Heat Loss by Helicity Injection in Spheromaks
}

\author{
T.K. Fowler
}

\begin{abstract}
A model is presented for spheromak buildup and decay including thermal diffusivity associated with magnetic turbulence during helicity injection. It is shown that heat loss by magnetic turbulence scales more favorably than gyroBohm transport. Thus gyroBohm scaling for the proposed ignition experiment would be the conservative choice, though present experiments may be dominated by magnetic turbulence. Because of a change in boundary conditions when the gun is turned off, the model may account for the observed increase in electron temperature in CTX after turnoff.
\end{abstract}

\section{INTRODUCTION}

In a previous paper ${ }^{1}$, we have shown that the highest electron temperatures achieved in the CTX spheromak ${ }^{2,3}$ could be explained as a quasi-steady state in which core ohmic heating balances a gyroBohm heat transport rate. If so, ohmic ignition would be possible in a device of modest size 1 . Heat loss due to magnetic turbulence was not considered.

In this paper, we will consider the effects of magnetic turbulence to be expected in association with Taylor relaxation. Enhanced losses of particles and energy due to turbulence have been observed in spheromaks $4,5,6$, and recent measurements in the MST (Wisconsin RFP) have contributed greatly toward quantifying the rates and toward identifying the loss processes with resistive MHD tearing modes 7,8. The MST measurements appear to agree qualitatively with the theory of Rechester and Rosenbluth ${ }^{9}$. Their theory predicts an electron thermal diffusivity in the collisionless limit of the form

$$
\chi_{\text {Mag }}=v_{e} L_{c} \frac{B_{1}^{2} X}{B_{o}^{2}}
$$


where $v_{e}$ is the electron thermal speed, $L_{c}$ is a correlation length along field lines, $B_{1 X}$ is the radial magnetic perturbation and $\mathrm{B}_{\mathrm{O}}$ is the unperturbed field.

As noted by Rechester and Rosenbluth ${ }^{9}$, Eq. (1) could lead to rapid electron heat transport even for tiny values of $B_{1 X}$. On the other hand, according to Taylor ${ }^{10}$, the spheromak should relax to a state with closed flux surfaces and $B_{1 X} \simeq 0$. Then any residual $B_{1 X}$ should be just that required to transport magnetic helicity and energy from surface to surface in order to maintain the Taylor profile against distortions away from this profile due to power input and ohmic decay. Here we are interested in what Taylor calls the Flux Core Spheromak (FCS $)^{10}$, in which external gun power builds up the spheromak field and sustains it in steady state. In the FCS, gun power is applied along a bundle of open-ended field lines along the axis of symmetry. Current driven on the open lines causes turbulence that drives helicity and power across the separatrix into the closed line confinement region encircling the flux core ${ }^{11}$.

\section{MODEL}

Following Bhattacharjee and Hameiri'2, we approximate the spheromak magnetic field evolution by

$$
\frac{\partial}{\partial t} \frac{B_{0}^{2}}{2 \mu}+\frac{1}{r} \frac{\partial}{\partial r} r P(r)=-(1+g) \eta j_{o}^{2}
$$

We describe heat flow by

$$
\frac{3}{2} \frac{\partial}{\partial t} n T_{e}-\frac{1}{r} \frac{\partial}{\partial r} r n\left(\chi_{m a g}+\chi_{G B}\right) \frac{\partial T_{e}}{\partial r}=\left(1+g_{e}\right) \eta j_{0}^{2}
$$

Here $\chi_{\mathrm{GB}}$ is the gyroBohm thermal diffusivity; $\eta$ is the Spitzer resistivity; $g$ is a reminder that both ions and electrons can be heated directly by damping of magnetic turbulence ${ }^{13}$; and $g_{e}$ is the portion of $g$ going to electrons, both directly and by ionelectron collisional energy transfer $\left(g_{e} \leq g\right)$.

Whereas in Ref. 12 the boundary condition in Eq. (2) was taken as $P=0$ at the plasma edge (appropriate for RFP's), for the flux core spheromak the boundary condition is

$$
(2 \pi a)^{2} P(a)=-f P_{\text {gun }}
$$


where $\mathrm{a}$ is the plasma minor radius and $\mathrm{fP}_{\mathrm{gun}}$ is the fraction of the gun power that propagates power and helicity from the flux core into the closed confinement region. When the gun is off, the boundary condition reverts to $P(a)=0$, so that Eq. (4) describes both gun on and gun off.

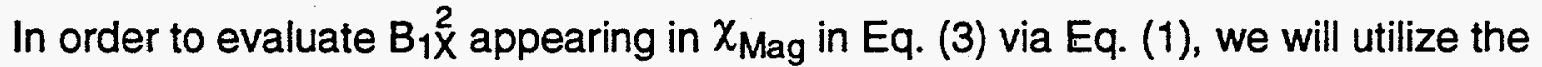
fact that we expect the system to hover near the Taylor state and use Eq. (2) to determine $\mathrm{B}_{1 X}^{2}$. To do so, we take

$$
\begin{aligned}
& B_{0}=B(t) f_{T}(r) \\
& j_{0}=\frac{\lambda}{\mu_{0}} B_{0}
\end{aligned}
$$

where $\lambda$ is the Taylor eigenvalue and $f_{T}(r)$ is the Taylor profile normalized to $f_{T}(a)=1$ so that $B(t)$ is the edge field as defined in Ref. 1 (i.e., $B$ at $r=R \simeq 2 a, z=0$ where $R$ is the radius of the flux conserver). Integrating Eq. (2) gives an equation for the magnitude $B(t)$,

$$
\frac{d E_{M A G}}{d t}=f P_{\text {gun }}-(1+g) P_{\Omega}(a)
$$

where, from Ref. 1, in MKS/keV units,

$$
E_{M A G}=\int \frac{B_{0}^{2}}{2 \mu_{0}}=1.7 \times 10^{6} B(t)^{2} R^{2} L
$$

and $P_{\Omega}(r)=\int_{0}^{r} \eta \dot{b}^{2}$, whereupon

$$
P_{\Omega}(a)=1.5 \times 10^{6} \frac{B(t)^{2}}{T_{0}^{3 / 2}} g_{\Omega}(a)
$$

Here $T_{0}$ is the peak electron temperature in the plasma core and $g_{\Omega}(a)$ is a factor that depends on the temperature profile. For a parabolic profile, in Ref. 1 we found $g_{\Omega}(a)=$ $2 C_{1}\left(T_{0} / T_{E}\right)^{1 / 2}$ where $T_{E}$ is the edge temperature and $C_{1}$ was taken as $C_{1}=0.5$ (see Section 7). 
To find $P(r)$ we integrate Eq. (2) partially, from $r$ to a, with the result

$$
(2 \pi)^{2} \text { ar } P(r)=-\frac{h(r)}{h(a)}\left[f P_{\text {gun }}-(1+g) P_{\Omega}(a)\right]-(1+g) P_{\Omega}(r)
$$

Here we have eliminated the $\partial \mathrm{B}_{0} \partial t$ term using Eqs. (4) and (6) and

$$
\int_{r}^{a} \frac{d}{d t} \frac{B_{0}^{2}}{2 \mu_{0}}=\frac{d E_{M A G}}{d t} \frac{(h(a)-h(r))}{h(a)}
$$

where $h(r)=\int_{0}^{r}$ rdr $f^{2}$. Note that Eq. (9) applies during buildup, decay and steady state.

Finally, to close the loop, we relate $P(r)$ to $B_{1}^{2}$ by

$$
P(r)= \pm X v_{A} \frac{B_{1}^{2}}{\mu_{0}}
$$

where $v_{A}$ is the Alfvén speed. Using Eqs. (1), (9) and (11)

$$
\chi_{M A G}=\frac{v_{e} L_{0} \mu_{0}}{v_{A} B_{o}^{2} X^{*}} P_{\Omega}(r) g_{p}(r)\left(1+g_{e}\right)
$$

with

$$
\begin{aligned}
X^{*} & =\frac{1+g_{e}}{1+g} X \\
& =\left|\frac{h(r)}{h(a)} \frac{f P_{\text {gun }}-(1+g) P_{\Omega}(a)}{(1+g) P_{\Omega}(r)}+1\right| \\
& =1, \quad, \text { gun on in steady state } \\
g_{p}(r) & =\left|\frac{h(r) P_{\Omega}(a)}{h(a) P_{\Omega}(r)}-1\right|, \text { gun off } .
\end{aligned}
$$

The choice of sign in Eq. (11) reflects the fact that, by Eq. (9), $P(r)$ is negative with the gun on [by which we mean $f P_{\text {gun }} \geq(1+g) P_{\Omega}(a)$ ] and $P(r)$ is generally positive when the 
gun is off. Physically, with the gun on, power is pumped inward from the flux core while during decay (gun off) power must be pumped from the decaying core field to the edge where it is needed to maintain the Taylor state.

From Eq. (12), we see that $\chi_{M A G} \propto P_{\Omega}(r)$, the ohmic dissipation. This is to be expected since $\chi_{M A G}=0$ in the Taylor state and the power input to maintain the field near the Taylor state is related to $P_{\Omega}$, by Eq. (6). From Eq. (11), the factor $X$ in the denominator of $\chi_{\text {MAG }}$ is a measure of the rate at which the Poynting flux distributes the input power over the profile. The slower the ohmic decay and the faster the redistribution of energy, the smaller the fluctuation level and hence the smaller is $\chi_{\text {MAG. }}$. Hence, $\chi_{\text {MAG }}$ is proportional to $P_{\Omega}$ but inversely proportional to $X$. These features of the model will be important in determining a conservative scaling law for spheromaks, in Section 5.

Beyond the assumptions stated above, the main physics of the model is embedded in $\mathrm{X}$, and in our selection of gyroBohm diffusion as the competing process. Given $\mathrm{X}$, Eqs. (3) and (6) determine the evolution of the system.

\section{QUALITATIVE FEATURES}

Before attempting to estimate $\mathrm{X}$, we first pause to note two qualitative features of the model.

First we consider the steady state with the gun on. Then dropping time derivatives, multiplying Eq. (2) by $\left(1+g_{e}\right) /(1+g)$ and adding this to Eq. (3) gives after integrating (treating $\mathrm{g}, \mathrm{ge}_{\mathrm{e}}$ as constants):

$$
\frac{1+g_{e}}{1+g} P(r)-n\left(\chi_{\text {mag }}+\chi_{G 0}\right) \frac{\partial T_{e}}{\partial r}=0
$$

For steady state $\mathrm{fP}_{\text {gun }}=(1+g) P_{\Omega}(a)$ and Eq. (9) gives $(2 \pi)^{2}$ ar $P(r)=-(1+g) P_{\Omega}(r)$. Then, neglecting $\chi_{\mathrm{GB}}$ for the moment and using Eqs. (1), (13) and (11) with the sign for the gun on, Eq. (14) becomes

$$
-X^{*} v_{A} \frac{B_{1 X}^{2}}{\mu_{0}}+\left(-n \frac{\partial T_{e}}{\partial r}\right) v_{A}\left(\beta_{e} \frac{m_{i}}{m_{e}}\right)^{1 / 2} L_{C} \frac{B_{1}^{2}}{B_{o}^{2}}=0
$$


Here we have used $v_{e}=v_{A}\left(\beta_{e} m_{j} / m_{e}\right)^{1 / 2}$ with $\beta_{e}=2 n T \mu_{o} / B_{o}^{2}$.

Treating $n, B_{o}$ and $X^{*}$ as constant, Eq. (15) becomes

$$
\frac{1}{2} \beta_{e}^{1 / 2} \frac{\partial \beta_{e}}{\partial r}=-\frac{1}{L_{c}}\left(\frac{m_{e}}{m_{i}}\right)^{1 / 2} X^{\star} .
$$

The solution averaged over the volume is

$$
\bar{\beta}_{e} \cong\left(\frac{m_{e}}{m_{i}}\right)^{1 / 3}\left(\frac{R X^{*}}{L_{C}}\right)^{2 / 3}
$$

Thus, we conclude that, if $\chi_{\text {MAG }}$ were the dominant loss process, ohmic heating produces a specific beta value (if $\mathrm{X}^{*} \sim$ constant). Indeed, RFP's do appear to reach a specific beta value, though the above may or may not be the explanation. Actually, we will find that $\mathrm{X}^{*}$. depends weakly on system parameters.

Next we consider the effect of turning off the gun. Integrating Eq. (3) over $0<r<a$ gives

$$
\frac{d E_{K E}}{d t}=-r n\left(\chi_{\text {mag }}+\chi_{G B}\right)\left(-\frac{\partial T_{e}}{\partial r}\right)_{r=a}+\left(1+g_{e}\right) P_{\Omega}(a)
$$

where $E_{K E}=\int 3 / 2 n T_{e}$ is the total electron kinetic energy. When the gun is turned off, $g_{p}(a)=0$ by Eq. (13) and hence $\chi_{M A G}(a)=0$ by Eq. (12). Hence EKE begins to increase. The temperature does rise after gun turnoff in CTX. Of course, the temperature would in any case continue to rise if steady state has not yet been reached at turnoff. We will return to these points in Section 7.

\section{TEARING MODES AND THE AVERAGE POYNTING VECTOR}

We now turn to a calculation of the coefficient $X$ in Eq. (11) for the resistive MHD tearing modes thought to be responsible for helicity propagation in RFP's and spheromaks ${ }^{14}$. 
We begin with

$$
\frac{\partial \vec{B}}{\partial t}=-\nabla \times \vec{E}=\nabla \times(\vec{v} \times \vec{B}-\eta \vec{j})
$$

where $\vec{E}$ satisfies Ohm's law,

$$
\vec{E}=-\vec{v} \times \vec{B}+\eta \vec{i}
$$

Time-averaging Eq. (20) gives

$$
\left.\vec{E}_{0}=-\overrightarrow{\langle v} \times \vec{B}\right\rangle+\eta \vec{J}_{0}
$$

where $\vec{E}$ is the externally applied field and $\overrightarrow{\langle\vec{V}} \times \vec{B}\rangle$ includes the MHD dynamo effect 12,14 . In a spheromak, $\vec{E}_{0}=0$ except in the flux core. Introducing Eq. (21) into the time-average of Eq. (19) and taking the scalar product with $\vec{B}_{0} / \mu_{0}$ gives

$$
\left.\frac{\partial}{\partial t} \frac{B_{0}^{2}}{2 \mu_{0}}=\frac{\vec{B}_{0}}{\mu_{0}} \cdot \nabla \times(\vec{v} \times \vec{B}\rangle-\eta \overrightarrow{j_{0}}\right)
$$

whence

$$
\left.\frac{\partial}{\partial t} \frac{B_{0}^{2}}{2 \mu_{0}}=\frac{\vec{B}_{0}}{\mu_{0}} \cdot \nabla \times(\vec{V} \times \vec{B}\rangle-\eta \overrightarrow{\mathrm{J}}^{2}\right)
$$

where in Eq. (23) we have used $\overrightarrow{\mathrm{j}_{0}} \times \overrightarrow{\mathrm{B}_{\mathrm{o}}} \cong 0$ for a Taylor state.

In comparing Eqs. (2) and (23), we see that, aside from the " $\mathrm{g}$ " term in Eq. (2), the Poynting vector is given by (slab approximation, $r \rightarrow x$ )

$$
-\frac{\partial P}{\partial x}=-B_{o y} \frac{\partial}{\partial x} F
$$




$$
F=\overrightarrow{\langle v} \times \vec{B}\rangle_{Z}=\underset{\vec{K}^{\prime}}{\sum} \vec{v} \vec{K}^{\prime} \times \vec{B}-\vec{k}^{\prime}
$$

using the notation of Refs. 12 and 15. Strauss has estimated F from quasi-linear and direct-interaction theories ${ }^{15}$, and Rutherford et al. have considered anomalous viscosity due to momentum transport corresponding to the $\chi$ in Eq. $(12)^{16,17}$. In all cases, $v_{1 X} \propto \gamma B_{1 X}$ where $\gamma$ is either the linear growth rate 18,19 or a non-linear rate due to turbulence $15,16,17$. Then $P$ takes the form

$$
P(r)=\left(\gamma \tau_{H} K\right) v_{A} B^{2} 1 x / \mu_{0}
$$

from which, by Eq. (11),

$$
\mathrm{X}=\left(\gamma \tau_{H} \mathrm{~K}\right)
$$

where $\tau_{H}=a / v_{A}, v_{A}$ being the Alfvén speed. The physics complexities have been buried in the dimensionless numbers $\mathrm{K}$ and $\mathrm{g}$. As an energy conservation equation, Eq. (2) is intended to include all non-linear processes associated with Taylor relaxation. Extrapolating from RFP Theory ${ }^{14}$, it is appropriate to identify the driving power $P(r)$ with the lowest-lying linearly unstable mode numbers. This power is distributed by inverse cascading to long wavelengths that drive the MHD dynamo and forward cascading to short wavelengths that dissipate as direct heating of ions and electrons ${ }^{11}$, represented here by the factors $\mathrm{g}$ and $\mathrm{ge}$.

In a strictly quasi-linear theory, $\mathrm{X}$ in Eq. (27) could be evaluated directly form linear theory 18,19 . From dimensional analysis, $X$ would then be a function of the Lundquist number $S$, given by

$$
\mathrm{S}=\frac{\tau_{\mathrm{R}}}{\tau_{H}}
$$

where $\tau_{R}=\left(a^{2} \mu_{0} / \eta\right)$. However, Rutherford 16,17 , Strauss 15 and others have found that non-linear corrections to $\gamma$ soon dominate, the most important being hyper-resistivity according to Strauss ${ }^{15}$, with

$$
\gamma \tau_{H} \equiv \frac{B_{1 X}}{B_{0}}
$$


and $K \cong 1$. Eq. (29) could have been guessed from the expectation that, at saturation, growth rates should scale as the square root of the specific free energy (here, $\mathrm{B}_{1} \mathrm{X}^{\left.2 / \mathrm{B}_{0}\right)^{2}}{ }^{20}$. Introducing Eq. (29) into Eq. (27) with $\mathrm{K}=1$ gives

$$
\mathrm{X}=\frac{\mathrm{B}_{1 \mathrm{X}}}{\mathrm{B}_{0}} .
$$

Given Eq. (30), we must solve Eqs. (6) and (9) to find $B_{1 X} / B_{0}$ (and $\left.X\right)$. However, without actually solving these equations, we can see that, roughly, Eq. (2) requires that in the plasma core

$$
(2 \pi a)^{2} P \equiv 2 \pi a \cdot \pi a^{2} \eta j_{o}^{2}=(2 \pi a)^{2} \frac{a}{\tau_{R}} \frac{B_{0}^{2}}{\mu_{o}}
$$

where $\tau_{R}$ is to be evaluated at the core temperature. Substituting Eq. (11) gives

$$
X \frac{B_{1} x^{2}}{B_{0}^{2}}=S^{-1},
$$

and, using Eq. (30),

$$
X=\frac{B_{1 X}}{B_{0}}=S^{-1 / 3} \text {. }
$$

This result was also obtained in Ref. 15.

The linear theory appears to give a higher value of $\mathrm{X}$ and hence Strauss' hyperresistive $X$ is the more conservative choice. For the linear theory $\gamma_{H} \propto S^{-3 / 5}$ and the resistive layer width $\delta=S^{-2 / 5} a^{18,19}$. Substituting the maximum value of $v_{X}$ (at $x=\delta$ ) into Eq. (25) gives $K \propto \delta^{-1} \propto S^{2 / 5}$, whereupon Eq. (27) yields $X \simeq S^{-1 / 5}$ [which exceeds $S^{-1 / 3}$ in Eq. (33)]. Other theories and experimental data in RFP's have suggested $B_{1 X} / B_{0} \simeq S^{-1 / 2}$ or $X=1$, which would be even more optimistic $20 A$.

Experimental values of $\beta$ and $X$ are compared with Theory in Table 1. Here the theoretical $\beta$ is given by Eq. (17) (with $\beta=2 \beta_{e}$ ) with $X$ given by Eq. (33). For simplicity, we take $g_{e}=0$. Since some of the data is in the collisional regime, it is necessary to correct $L_{c}$ for collisional effects, as discussed by Krommers, Oberman 
TABLE 1

Comparison of experimental vlaues of $\beta$ and $X$ with Theory,

for data from Ref. 23

\begin{tabular}{|c|c|c|c|c|c|c|c|c|c|c|}
\hline Experiment* & $\begin{array}{c}\mathrm{T} \\
(\mathrm{keV})\end{array}$ & $\begin{array}{c}n \\
\left(10^{20} m^{-3}\right)\end{array}$ & $\begin{array}{c}\text { B } \\
\text { (Tesla) }\end{array}$ & $S$ & $\frac{R}{\lambda_{e}}$ & $\beta=2 \beta_{e}$ & $\beta$ Theory & $\mathrm{X}$ & $\begin{array}{c}\mathrm{X}=\mathrm{S}-1 / 3 \\
\text { Theory }\end{array}$ & $\left(\frac{-\ln x}{\ln S}\right)$ \\
\hline S-1 Low $T_{e}$ & 0.02 & 0.8 & 0.15 & $5.0 \times 10^{3}$ & 18.0 & 0.06 & 0.17 & 0.03 & $\overline{0.06}$ & 0.4 \\
\hline S-1 High $T_{e}$ & 0.05 & 0.8 & 0.29 & $3.8 \times 10^{4}$ & 2.9 & 0.037 & 0.04 & 0.08 & 0.03 & 0.24 \\
\hline CTX 100 I & 0.1 & 3.9 & 1.19 & $2.2 \times 10^{5}$ & 1.96 & 0.02 & 0.021 & 0.05 & 0.017 & 0.36 \\
\hline CTX 100 II & 0.1 & 1.1 & 0.72 & $2.5 \times 10^{5}$ & 0.55 & 0.017 & 0.0135 & 0.06 & 0.016 & 0.23 \\
\hline CTX $200 \mathrm{I}$ & 0.2 & 3.9 & 1.23 & $6.5 \times 10^{5}$ & 0.49 & 0.041 & 0.011 & 0.24 & 0.011 & 0.11 \\
\hline CTX $200 \mathrm{II}$ & 0.2 & 1.1 & 0.74 & $7.3 \times 10^{5}$ & 0.14 & 0.032 & 0.009 & 0.21 & 0.011 & 0.11 \\
\hline CTX $400 \mathrm{I}$ & 0.4 & 3.9 & 1.23 & $1.8 \times 10^{6}$ & 0.12 & 0.083 & 0.007 & 0.90 & 0.008 & 0.007 \\
\hline CTX $400 \mathrm{II}$ & 0.4 & 1.1 & 0.75 & $2.1 \times 10^{6}$ & 0.035 & 0.063 & 0.007 & 0.64 & 0.008 & 0.03 \\
\hline
\end{tabular}

"Notation in Ref. 23. 
and Kleva21. Here we have taken the simple correction proposed by Rechester and Rosenbluth ${ }^{9}$, namely, $L_{c}=R\left(1+R / \lambda_{e}\right)^{-1}$ where $\lambda_{e}$ is the electron mean-free-path. The "experimental" value of $X$ is found by solving Eq. (17) for $X$ with the experimental value of $\beta$ and the above formula for $L_{c}$. Also shown is $(-\ln X / \ln S)$, which should be 0.33 according to Eq. (33) (or 0.2 for the linear estimate or zero if $B_{1 X} / B_{0}=S^{-1 / 2}$ ). Agreement with the model is only qualitative. Experimental values of $\beta$ appear to exceed the theoretical values in the collisionless regime $\left(\lambda_{e}>R\right)$, a favorable result if true.

\section{SCALING TO IGNITION}

Part of the material summarized in this section appears also in Ref. 22. Following Ref. 1, we can now develop a scaling law for the temperature achieved by ohmic heating if $\chi_{\text {mag }}$ (rather than gyroBohm) is the heat loss mechanism. Again we take $n=n_{\min }$ given by ${ }^{1}$

$$
n_{\min }=\frac{1.2 \times 10^{20} B}{R \sqrt{T_{i}}}
$$

and we equate core ohmic heating to the loss ${ }^{1}$,

$$
P_{\text {core }}=\frac{10^{6} B^{2} R}{T_{e}^{3 / 2}}\left(1+g_{e}\right)=(2 \pi a)^{2} \times \frac{n T}{a} .
$$

With $n=n$ MIN and $T=T_{i}=T_{e}$, this gives (aside from constants) ${ }^{21}$

$$
T^{2}\left(\frac{\chi}{1+g_{e}}\right)=B R
$$

This scaling describes ohmic heating for any $\chi$.

As was shown in Ref. 1 , with gyroBohm scaling, $\chi_{G B} \propto T^{3 / 2} / B^{2} R$, Eq. (36) gives

$$
T_{G B}=0.7 B^{6 / 7} R^{4 / 7}
$$

where the coefficient was chosen to fit CTX data22. To obtain a corresponding scaling

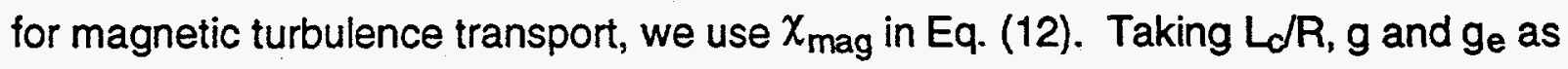
constant, $n=n_{M I N}$ in $v_{A}$, and $X$ in Eq. (33), Eq. (12) gives 


$$
x_{\operatorname{mag}} \propto \frac{v_{e} L_{C} P_{\Omega}}{v_{A} B_{0} r a X} \propto T^{-2 / 3} B^{-1 / 3}
$$

Then, combining Eqs. (36) and (38) gives as a scaling for Rechester-Rosenbluth transport,

$$
\mathrm{T}_{\mathrm{RR}}=0.9 \mathrm{~B} \mathrm{R}^{3 / 4} \text {, }
$$

where again the coefficient is obtained by fitting CTX data22. This coefficient implies $L_{c} \ll R$. For $L_{c}=R$, a better fit is obtained with $B_{1} \times / B_{0}=S^{-1 / 2}$, giving $T_{R R} \propto B^{2} R^{2}$ (very optimistic) 22 .

The scalings in Eqs. (37) and (39) apply only to ohmic heating in the collisionless regime (appropriate to future experiments and marginally appropriate for the highest temperatures achieved in CTX). In the collisional regime, $L_{c}$ must be reduced as discussed in Section 4. A smaller $L_{c}$ allows access to higher beta by ohmic heating, according to Eq. (17). On the other hand, auxiliary heating (not subject to the above scaling laws) provides access to higher beta values in the more relevant collisionless regime.

In Ref. 22, it is noted that the gyroBohm scaling in Eq. (37) gives lower values of $T$ than does the Rechester-Rosenbluth scaling. Thus the gyroBohm scaling assumed in Ref. 1 should be a more conservative basis for extrapolating to ignition.

Finally we note that, whatever the appropriate coefficients in Eqs. (37) and (39), eventually the gyroBohm scaling would dominate above a critical value of the field given by

$$
B_{\text {crit }}=C R^{-5 / 4}
$$

In principle, gyroBohm applies if $B>B_{\text {crit, }}$ while the Rechester-Rosenbluth scaling applies if $B<B_{\text {crit. }}$ However, because these scalings are very nearly the same, the coefficient $C$ is very sensitive to assumptions. A critical condition of the form in Eq. (40) obtains for $X=S^{-x}$, any $X$. 


\section{DIRECT WAVE HEATING}

In Ref. 13, it is shown that departures from the Taylor profile provide excess free energy that directly heats particles by wave damping. The extent of departure from the Taylor state to be expected is not easily calculated, and we have in fact assumed the Taylor state as a way of estimating fluctuation levels in previous sections. However, the observation that $T_{i}>T_{e}$ in both RFP's ${ }^{13}$ and spheromaks ${ }^{23}$ infers the reality of wave heating.

Direct heating of the ions would be harmful if energy imparted to the ions is lost directly, by charge exchange ${ }^{6}$ or by turbulence. This is illustrated in Eq. (13) where a large value of $g$ but a small value of $g_{e}$ acts to reduce $X^{*}$, implying an increase in the fluctuation level that increases $\chi_{\text {mag }}$ with no compensatory heating of the electrons. Such effects would increase somewhat the field required to reach ignition. Otherwise, direct heating that ultimately heats the electrons is only beneficial, especially if such heating scales more favorably with $T_{e}$ than does ohmic heating.

The collisional heating of electrons by the ions can conveniently be scaled to ohmic heating by expressing $n$ in terms of $n_{\min }$ where $j_{0}=n_{\min } e v_{i}$. Then, if $n \tau_{e}=10^{18} T_{e}{ }^{3 / 2}$ is the ion-electron collisional energy transfer rate, the ion-electron heating power is

$$
\begin{aligned}
P_{i e} & =\frac{3}{2} n \frac{T_{i}-T_{e}}{\tau_{i e}}=\frac{n^{2}}{n^{2} \min }\left(1-\frac{T_{e}}{T_{i}}\right) \frac{m_{i}}{2 e^{2}} \frac{1}{\eta\left(n \tau_{i e}\right)} \eta j_{o}^{2} \\
& =2.5 \frac{n^{2}}{n^{2} \min }\left|1-\frac{T_{e}}{T_{i}}\right| \eta j_{o}^{2} .
\end{aligned}
$$

For CTX parameters ${ }^{23}$, typically $T_{e}=0.4 T_{e}$ and $n=n_{\min }$, from which we conclude that ion heating is about 1.5 times the ohmic heating. If this heating is derived from wave heating of the ions, and if wave heating equipartitions between ions and electrons as estimated in Ref. 13, the total non-ohmic heating is 3 times ohmic, or $g_{e}=3$ in Eq. (3) for CTX. Then, from Eq. (36), the $\chi$ value implied by fitting CTX temperature data is 4 times larger and $\tau_{E}$ is 4 times smaller than our earlier estimates 1,21 . 


\section{BEHAVIOR AFTER GUN TURNOFF}

In Section 3, it was noted that our model predicts a drop in $\chi_{\text {mag }}$ at the edge $(r=a)$ when the gun is turned off, and that this abrupt change in $\chi$ may account for an observed increase in $T_{e}$ after the gun was turned off in CTX. Understanding the behavior after turnoff is important in scaling present experimental results to larger devices.

The transient behavior predicted by the model requires computer calculations not yet attempted. We can make some progress analytically by noting that, even though $B_{0}$ decays, an initial increase in ( $\mathrm{nT}_{e}$ ) would be followed by an ultimate decay so that $\partial\left(n T_{e}\right) / \partial t=0$ at some time after gun turnoff. At that instant, dropping $\partial T_{e} / \partial t$ and integrating gives

$$
-(2 \pi)^{2} \operatorname{arn}\left(\chi_{\text {mag }}+\chi_{G B}\right) \frac{\partial T_{e}}{\partial r}=\left(1+g_{e}\right) P_{\Omega}(r)
$$

The boundary condition is $T=T_{E}$ at $r=a$; Eq. (51) already generates $\partial T_{e} / \partial r=0$ at $r=$ 0 if $\chi_{G B}=0$. Note that Eq. (14), in which both $\partial\left(n T_{e}\right) / \partial t$ and $\partial B_{o} / \partial t$ are zero, does not apply to the transient phase, but Eq. (41) applies both to steady state and at any time that $\partial\left(n T_{e}\right) / \partial t$ happens to be zero. A flaw in this reasoning concerns the possibility that there is a significant delay in reaching $\partial\left(n T_{e}\right) / \partial t=0$ at different radii. And of course we will not know $B_{0}$ at that time, except by measurement, unless the complete transient problem is solved. Finally, we have not succeeded in determining whether or not solutions of Eq. (42) are multi-valued or unique.

The interesting features concerning turnoff are contained in $\mathrm{gp}_{\mathrm{p}}(r)$, Eq. (13). Since $T_{e}(r) \simeq T_{0}$ near $r=0$,

$$
P_{\Omega}(r)=\frac{r^{2}}{a^{2}} P_{\Omega o} g_{\Omega}(r)
$$

and, taking $B_{o}$ as constant, $h(r) / h(a)=r^{2} / a^{2}$, we obtain

$$
g_{p}(r)=\left|\frac{g_{\Omega}(a)}{g_{\Omega}(r)}-1\right|
$$


where

$$
g_{\Omega}(r)=\frac{1}{r^{2}} \int_{0}^{r} 2 r d r\left(\frac{T_{0}}{T_{e}(r)}\right)^{3 / 2}
$$

Here $T_{0}=T_{e}(0)$ and, from Ref. 1 ,

$$
P_{\Omega 0}=1.5 \times 10^{6} \frac{B_{0}^{2} R}{T_{0}^{3 / 2}}
$$

Again, we see that, since $g_{p}(a)=0$ after turnoff by Eq. (46), $\chi_{\text {mag }}(a)=0$ after turnoff [and, from Eq. (43), $\chi_{\text {mag }}(0)=0$ both before and after turnoff]. Thus, after turnoff, $\chi_{\text {mag }}$ can only dominate over a domain $0<r_{1}<r<r_{2}<a$. On the other hand, since after turnoff core energy must be transported to the edge in order to maintain the Taylor state, $\chi_{\text {mag }}$ may actually increase in magnitude after turnoff. In the model, this would be reflected in $g_{\Omega}(a)>g_{\Omega}(r)$ so that $g_{p}(r)>1$ in the core after turnoff.

To see whether or not $\mathrm{g}_{\mathrm{p}}>1$, let us consider possible solutions of Eq. (42). From Eqs. (42), (51) and (52), we would find

$$
x_{\operatorname{mag}} \propto \frac{T_{e}(r)}{T_{0}}
$$

while for gyroBohm diffusion

$$
\chi_{\mathrm{GB}} \propto\left(\frac{T_{e}(r)}{T_{0}}\right)^{3 / 2} .
$$

Thus we expect solutions of the form

$$
\frac{T_{e}(r)}{T_{0}}=\left(1-f_{T}(r)\right)^{\alpha}
$$

where $f_{T}(0)=0$ and $\alpha=1 / 2$ or $2 / 5$ depending on whether $\chi_{\text {mag }}$ or $\chi_{G B}$ is dominant. For example, to leading order let $f_{T}(r)=f\left(r^{2} / a^{2}\right)$ with

$$
f=1-\left|\frac{T_{E}}{T_{0}}\right|^{1 / \alpha} \text {. }
$$


Then for $T_{E}<<T_{0}$,

$$
g_{\Omega}(r)=\frac{a^{2}}{r^{2}} \frac{1}{\beta} \frac{1}{f}\left[1-\left(1-f \frac{r^{2}}{a^{2}}\right)^{\beta}\right] \cong \frac{a^{2}}{r^{2}} \frac{1}{\beta}\left[1-\left(1-\frac{r^{2}}{a^{2}}\right)^{\beta}\right]
$$

where $\beta=1-3 / 2 \alpha=1 / 4$ for $\alpha=1 / 2$ (magnetic turbulence) and $\beta=2 / 5$ for $\alpha=2 / 5$ (gyroBohm). Note that, whereas we found $g_{\Omega}(a) \propto\left(T_{d} / T_{E}\right)^{1 / 2}$ for $\alpha=1$ in Ref. 1, here $\mathrm{g}_{\Omega}$ is independent of the edge temperature (the change of behavior occurring at $\alpha=2 / 3$ ). For values $T_{0} / T_{E}=0.1$ typical in present experiments, $g_{\Omega}(a)=2.5$ to 6 for all cases, $1 / 2<\alpha<1$. Other factors that could flatten the profile at the edge and increase $g_{\Omega}(a)$ are field error and gas penetration inside the separatrix.

From Eq. (51), we see that $g_{\Omega}(r)$ increases monotonically from 1 to $\beta^{-1}$, whereupon $g_{p}(r)$ in Eq. (44) decreases monotonically from $\beta^{-1}$ to zero, and $\chi_{\text {mag }}$ has a broad maximum between $0<\mathrm{r}<\mathrm{a}$ where $\mathrm{g}_{\mathrm{p}}>1$ and $\chi_{\text {mag }}$ after turnoff exceeds its value with the gun on, as anticipated above. This tends to flatten $T_{e}(r)$ which terminates in a steep "pedestal" where $\chi_{\text {mag }} \sim 0$ and $\chi_{G B}$ dominates. Thus, recalling that the above discussion concerns the quasi-steady state that exists when $T_{e}$ reaches its maximum value after turnoff $\left(\partial \mathrm{T}_{e} / \partial \mathrm{t}=0\right)$, we conclude that the maximum temperature achieved reflects a complicated mix of magnetic turbulence and gyroBohm scaling. Hence, fitting gyroBohm scaling to the data after turnoff, as was done in Ref. 21 and Section 5, is pessimistic since in effect gyroBohm applies only in the steep edge as if the radius were much smaller than a. Further discussion of gyroBohm scaling is given in Appendix A.

Based on the above arguments, in Table 2 we present a revised buildup scenario for the ignition experiment that continues to assume gyroBohm scaling by Eq. (37), as in Ref. 1, but revised estimates of the required gun power based on Eq. (51). 
Table 2

Revised Buildup Scenario for the Ohmic Ignition Experiment $(R=1.5 \mathrm{~m})$

B $\quad T \quad$ Gun Power(1)

(T) (keV)

to offset ohmic loss

(MW)

Gun Power
to build
up $B_{\circ}$
(MW)

PCoil(2)

(MW)
PTotal

(MW)

Ohmic Buildup

$\begin{array}{cccccc}0.5 & 0.5 & 11-26 & 40 & 0.4 & 51-66 \\ 1.0 & 0.9 & 16-38 & 40(3) & 2 & 58-80 \\ 3.0 & 2.2 & 37-88 & 40(3) & 15 & 92-143 \\ 6.2 & 4.2 & 60-143 & 40 & 65 & 165-248\end{array}$

Ignited burn(4)

$8.6 \quad 20$

$11-26$

0

125

136-151

(1) For $f P_{\text {gun }}=P_{\Omega}(a)+d E M a g / d t$ with $P_{\Omega}$ (a) given by Eq. (42). The range of values corresponds to $g_{\Omega}(a)=3, f=.5$ at the lower end and $g_{\Omega}(a)=10, f=.7$ at the upper end.

(2) $P_{\text {coil }}$ from Table 1, Ref. 1. Note error. Should be $B^{2} L$ (not $B L^{2}$ ).

(3) It may be better to increase power here and decrease it earlier and later.

(4) From Ref. 1, where $P_{\text {gun }}=26 \mathrm{MW} /$ based on $P_{\Omega}(a)$ in Ref. 1. 


\section{DISCUSSION}

The model presented here appears to be sufficiently promising to become the basis for developing a computer code to aid experimental data analysis and machine design. Several qualitative features have emerged:

(1) Since maintaining the Taylor state is primarily a competition between injected power and classical resistivity, transport due to fluctuations required to maintain the Taylor state exhibits a classical scaling with temperature $\left(\propto \mathrm{P}_{\Omega}\right)$ that is more favorable than gyroBohm scaling. As a consequence, the use of gyroBohm scaling to design an ohmic ignition experiment, as in Ref. 1 , is arguably a conservative approach.

(2) The combination of ohmic heating and magnetic turbulence heat losses would lead to a limiting value of $\beta_{e}$ in steady state that depends only weakly on plasma parameters. This value of $\beta_{e}$ could be exceeded using auxiliary heating, or possibly by direct damping of waves (the factors $g$ and $g_{e}$ in Section 2). Thus experiments with auxiliary heating could be used to explore the actual instability-based limits on beta in steady state or during decay.

(3) A new look at ohmic losses shows that the total ohmic loss $\mathrm{P}_{\Omega}$ (a) may or may not be sensitive to the edge temperature, depending on the steepness of the profile. For example, temperature profiles to be expected either with magnetic turbulence or gyroBohm diffusion are steeper than that assumed in Ref. 1. Consequently, though enhanced by the edge, with these profiles, the total ohmic loss that must be overcome by gun power would no longer be sensitive to the edge temperature set by the open-ended flux core or scrape off layer. A new buildup scenario based on these observations is given in Table 2. Any other processes (gas penetration, field error, etc.) that flatten $T_{e}$ at the edge would again enhance the ohmic loss as the system tries to restore the Taylor state.

(4) The role of magnetic turbulence is quite different with the gun on or off. When the gun is on, turbulence transmits helicity and power inward across the magnetic surface separating the open-ended flux core from the closed confinement region. This results in heat loss from the closed lines to the flux core (and the wall or SOL). When the gun is turned off, this edge turbulence goes away and turbulent helicity transport only occurs internally as needed to 
maintain the Taylor state. This latter situation, after turn off, is similar to that in an RFP in which fluctuations are strong in the interior but weak in the edge. Then some other process (gyroBohm diffusion in our model) determines the heat confinement in the edge. An important difference between RFP's and spheromaks in this respect is that, while the quiet zone is well defined in RFP's (the reversal layer), "quietness" is a more gradual property in spheromaks determined by a competition between transport properties. In this sense, in present day spheromaks for which $B<B_{\text {crit, }}$, the best information about the non-tearing processes that will dominate large devices would be contained in the sharp temperature gradient that would be expected to develop at the edge after gun turnoff.

\section{ACKNOWLEDGMENT}

The author gratefully acknowledges helpful discussions with Nathan Mattor, Jim Hammer, Bick Hooper and Alain Brizard.

Work performed under the auspices of the U.S. Department of Energy by Lawrence Livermore National Laboratory under Contract No. W-7405-Eng-48. 


\section{APPENDIX A}

As was discussed in Section 5, the gyroBohm scaling employed in designing the ohmic ignition facility in Ref. 1 was obtained by fitting CTX data after gun turnoff when the highest electron temperatures were achieved. In Section 7, we found that indeed gyroBohm diffusion (or some other non-tearing process) should dominate in the edge region after turnoff. However, as noted in Section 8 , Item (4), magnetic turbulence scaling and gyroBohm scaling would be co-mingled in a complicated way even in the edge region, and turbulence probably dominates the interior. Thus, while the actual gyroBohm scaling would be at least as favorable as the result we obtained from CTX, gyroBohm (or other) processes may actually be weaker than is implied by our scaling in Eq. (37); that is, the true coefficient in this formula may be greater than 0.7 .

In Ref. 1, we actually attempted to calculate the coefficient in Eq. (37) based on work of Covaliu24. The value 0.7 , which fits the data fairly well as shown in Table 1 , is also very close to the value we obtained by scaling Covaliu's results that provide a good fit ( $20 \%$ accuracy) to a wide range of tokamak data24. However, the questions raised above have prompted me to revisit my scaling from Covaliu's work in which only scaling with the safety factor $q$ was taken into account.

As was discussed in Ref. 1, Covaliu employed a gyroBohm formula for trapped electron modes due to Tang 25 ,

$$
\chi_{G B}=\frac{5 T_{e}^{2}}{e^{2} B_{o}^{2} v_{e}}\left[\frac{q A a}{L_{N} L T}+q\left(\frac{1}{L_{N}}+\frac{1}{L_{T}}\right)\right]
$$

where $A$ is the aspect ratio and $L_{N}$ and $L_{T}$ are logarithmic scale factors for density and temperature. His result for tokamaks can be recovered approximately by keeping only the first term and solving

$$
-\frac{1}{r} \frac{\partial}{\partial r} r n \chi_{G B} \frac{\partial T}{\partial r}=H
$$

which becomes, after one integration and some algebra,

$$
y^{1 / 2}\left(\frac{d y}{d x}\right)^{2}=\frac{Q}{q A}
$$




$$
\begin{aligned}
& Q=\frac{\mathrm{Ha}^{2}}{4 \mathrm{n}_{0} \chi_{0} T_{0}} \\
& \chi_{0}=\frac{5 T_{0}^{2}}{\mathrm{e}^{2} \mathrm{~B}^{2} v_{\mathrm{eo}} \mathrm{a}} .
\end{aligned}
$$

Here $H$ is taken as constant, $n=n_{0}\left(1-x^{2}\right)$ with $x=r / a$, and $y=T_{e}(r) / T_{0}$ normalized to $y(0)=1$. Taking the square root of Eq. (A3) (with negative sign) and solving gives

$$
y=\frac{5}{4}\left(\frac{Q}{q A}\right)^{1 / 2}(1-x)^{4 / 5}
$$

Then setting $y(0)=1$ determines $Q$ as

$$
Q=\frac{16}{25} q A \text {. }
$$

Since $Q$ and $H$ determine $T_{0}$ from Eq. (A4), the essential information is contained in $Q$. In Covaliu's work, $q$ is not constant, the average value being $\bar{q}=2$. In Ref. 1 , we used this fact to scale his results while ignoring other geometric differences between tokamaks and spheromaks that enter his results in a complicated manner. However, our calculation above displays the geometric effects in simple form. For $A=3$ typical of tokamaks (and assumed by Covaliu), Eq. (A7) gives $Q=3.84$ to 5.76 for $q=2$ to $q$ $=3$. His result is $Q=5.0$, which yields a good fit to $\tau_{E}$ for tokamaks as already noted.

One can either use $Q$ to find $\tau_{E}=H^{-1} \int_{\frac{3}{2}} n T_{e}$ as Covaliu did (and as we did for spheromaks in Ref. 1) or one can obtain the temperature scaling analogous to Eq. (37) directly by taking $2 \pi^{2} \mathrm{a}^{3} \mathrm{H}=\mathrm{P}_{\text {core }}$ given by Eq. (35). Taking the latter approach and using Eq. (A4) with $n=n_{M i n}$ in Eq. (36) gives

$$
T_{G B}=1.89 B^{6 / 7} R^{4 / 7} \text {. }
$$

A similar result would be obtained if we set $L_{N}=\infty$ and keep only the last term in Eq. (A1).

The reason for the larger coefficient in Eq. (A8) as compared to Eq. (37) is the more transparent scaling with $q$ and $A$. The coefficient in Eq. (A8) is proportional to (qA) $2 / 7$. Thus scaling from tokamaks, with $q A=6$ to 9 , to spheromaks, with $q A=0.5$, introduces a multiplier $=3$, which is much greater than the correction applied in Ref. 1 . 
While the actual value calculated in $\mathrm{Eq}$. (A8) cannot be taken too seriously, the calculation does demonstrate the need to determine more accurately the electron gyroBohm rate to be expected in spheromaks. If Eq. (A8) were actually correct, present ohmic heating experiments are dominated by magnetic turbulence and the critical field at which gyroBohm scaling would dominate with ohmic heating becomes very large $\left[B_{\text {crit }}=180 R^{-5 / 4}\right.$, by equating Eqs. (39) and (AB)]. Hence the somewhat more favorable scaling of Eq. (39) would apply in the ohmic phase of the ignition buildup scenario. 


\section{References}

1. T.K. Fowler, "A Spheromak Ignition Experiment Reusing Mirror Fusion Test Facility (MFTF) Equipment," UCRL-ID-114696, September 28, 1993.

2. F.J. Wysocki, J.C. Fernandez, I. Hennins, T.R. Jarboe, and G.J. Marblin, Phys. Rev. Letters $\underline{65}, 40$ (1990).

3. T.R. Jarboe, F.J. Wysocki, J.C. Fernandez, I. Hennins, and E.J. Marblin, Phys. Fluids B, 2, 1342 (1990).

4. R.M. Mayo, C.K. Choi, F.M. Levinton, A. Janos, and M. Yamada, Phys. Fluids B 2, 115 (1990).

5. J.C. Fernandez, C.W. Barnes and T.R. Jarboe, et all., Nucl. Fusion 28, 1555 (1988).

6. R.M. Mayo, F.M. Levinton, D.D. Meyerhofer, T.K. Chu, S.F. Paul, and M. Yamada, Nucl. Fusion 29, 1493 (1989).

7. E. Fiksel, S.C. Prager, W. Shen, and M. Stoneking, "Measurement of Magnetic Fluctuation Induced Energy Transport," submitted to Phys Rev. Letters.

8. M.R. Stoneking, S.A. Hokin, S.C. Prager, G. Fiksel, and H.J. , "article Transport Due to Magnetic Fluctuations," in preparation.

9. A.B. Rechester and M.N. Rosenbluth, Phys. Rev. Letters 40, 38 (1978).

10. J.B. Taylor, Review of Mod. Phys. $\underline{58,} 341$ (1986).

11. J.B. Taylor and M.F. Turner, Nuclear Fusion 29, 219 (1989).

12. A. Bhattacharjee and E. Hameiri, Phys. Rev. Letters 57, 206 (1986).

13. N. Mattor, P.W. Terry, and S.C. Prager, "Anomalous lon Heating From the Dynamo in a Reversed Field Pinch," Comments Plasma Phys. Controlled Fusion, 15, 65 (1992).

14. D.D. Schnack, "Magnetohydrodynamic Theory of the Reversed-Field Pinch Dynamo," ISPP-8 "Piero Caldirola," Physics of Alternative Magnetic Confinement Schemes, S. Ortolani and E. Sindoni, Eds., SIF, Bologna, 1991.

15. H.R. Strauss, Phys. Fluids 29,3668 (1986). See also, Z. Yoshida, H.R. Strauss and E. Hameiri, "Hall Effects on Anomachrous Heat, Particle and Helicity Transports Through Tearing-Mode Turbulence", New York University, DOE/ER53223-158, MF-122, January 1991.

16. H.P. Furth, P.H. Rutherford and H. Selberg, Phys. Fluids 16, 1054 (1973).

17. P.K. Kaw, E.J. Valeo and P.H. Rutherford, Phys. Rev. Letters 43,1398 (1979).

18. M.N. Rosenbluth and P.H. Rutherford, Chapter 2, Fusion Vol. 1, Part A, E. Teller, Editor, Academic Press, New York, 1981.

19. H.P. Furth, J. Killeen and M.N. Rosenbluth, Phys. Fluids 6, 459 (1963).

20. T.K. Fowler, "Thermodynamics of Unstable Plasmas" Advances in Plasma Physics, A. Simon and W.B. Thompson, Eds., Interscience, New York, 1968. 
20 A R.J. LaHaye, T.N. Carlstrom, R.R. Goforth, G.L. Jackson, M.J. Shaffer, T. Tamano, and P.L. Taylor, Phys. Rev. Letters, 27, 2576 (1984).

21. J.A. Krommes, C. Oberman and R.G. Kleva, J. Plas. Phys. 30, 11 (1983.)

22. T.K. Fowler, J. Hardwick, and T.R. Jarboe, "On The Possibility of Ohmic Ignition in a Spheromak, "February 1994, submitted to Comments on Plas.

Phys. and Contr. Fusion.

23. R.M. Mayo, D.J. Hurlburt and J.C. Fernandez, Phys. Fluids B $\underline{5}, 4002$ (1993).

24. Z. Covaliu, "Sensitivity of a Tokamak Heat Transport Model to Edge Effects and Density Profile," UC-BFE-013, June 18, 1990.

25. W.M. Tang, "Microinstability Based Model for Anomalous Thermal Confinement in Tokamaks," Princeton report PPPL-2311, 1986. 